\title{
Procedimento de intervenção aplicado a cuidadores e pacientes relacionado à comunicação em consultas de acompanhamento em câncer infantil
}

\author{
Marina Kohlsdorf \\ Áderson Luiz Costa Junior
}

\section{RESUMO}

Constitui como objetivo deste trabalho analisar um procedimento de intervenção, aplicado a cuidadores e pacientes, , relacionado à comunicação em consultas de acompanhamento em câncer infantil. Participaram do estudo 15 díades criança-cuidador, que foram individualmente entrevistadas antes de consultas para tratamento de câncer infantil. Nessa entrevista, denominada pré-consulta, cada díade listava dúvidas, dificuldades, demandas e assuntos para serem discutidos na consulta subsequente. Esses temas eram registrados em um protocolo de pré-consulta, o qual anexado ao prontuário da criança, para servir como lembrete ao médico na consulta em seguida. Os resultados mostraram quantidades variadas de itens listados ao longo do procedimento, dado possivelmente relacionado a demandas distintas em função de diferentes etapas do tratamento. Os assuntos estiveram concentrados em cuidados, alimentação e fundamentos da neoplasia; houve dúvidas que, mesmo discutidas em consultas, permaneceram presentes em atendimentos subsequentes. Este estudo fornece subsídios para outras pesquisas e intervenções sobre comunicação em oncologia pediátrica.

Palavras-chave: câncer em crianças; relação médico-paciente; pediatria; intervenção psicoterapêutica.

\section{ABSTRACT \\ Intervention procedure applied to caregivers and patients related to communi- cation in follow-up appointments in pediatric cancer}

This paper aimed to analyze an intervention procedure, applied to caregivers and patients, about communication in follow-up appointments on childhood cancer. In this study, 15 childcaregiver dyads took part, and they were individually interviewed before medical visits in childhood cancer. During this pre-appointment interview, each dyad would list doubts, difficulties, demands, and issues to be discussed in the next appointment. These themes were registered in the pre-appointment protocol, attached to the child's medical record, as a memo to the pediatrician in the following medical visit. Results show unbalanced amounts of listed items along the procedure, data that may be related to special demands regarding different treatment stages. The themes were focused on self-care, food and biological cancer aspects; there were doubts that even when discussed in medical visits remained in subsequent appointments. This study provides subsidies to other researches and interventions regarding communication in pediatric settings.

Keywords: cancer in children; physician-patient relationship; pediatrics; psychotherapeutic intervention.

Nas últimas três décadas, houve importante avanço na atenção à população pediátrica com consequente melhora nos indicadores de morbimortalidade desta população, progresso conseguido devido em grande parte à melhor eficiência da comunicação durante consultas, que as quais representam um contexto primordial para a promoção do desenvolvimento ao possibilitar a abordagem precoce de temas como cuidados, proteção e fatores psicossociais

\section{Sobre os Autores}

M. K.

orcid.org/0000-0002-7029-3270

Centro Universitário de Brasília

(UniCeub) - Brasília, DF

jmarinak@unb.br

\section{A. L. C. J.}

orcid.org/0000-0002-8343-0043 Universidade de Brasília (UnB) Brasília, DF

aderson@unb.br

\section{Direitos Autorais}

Este é um artigo de acesso aberto e pode ser reproduzido livremente, distribuído, transmitido ou modificado, por qualquer pessoa desde que usado sem fins comerciais. 0 trabalho é disponibilizado sob a licença Creative Commons CCBY-NC.

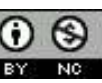




\section{H NTERAC̄öEM IF PSICOLOGIA}

condicionantes ao processo saúde-doença (Forrest et al., 2012).

A comunicação corresponde a um processo complexo e multidimensional, embasado em fatores culturais e sociohistóricos, em que informações e sua compreensão são compartilhadas entre pessoas a partir de códigos comuns (Fisher \& Broome, 2011; Forrest et al., 2012). Além da satisfação dos usuários, a comunicação em contexto pediátrico influencia adesão aos cuidados, sintomas e respostas clínicas, compreensão sobre diagnóstico e tratamento, manejo de fatores psicossociais relacionados à terapêutica, melhor recordação das explicações e menos retornos ambulatoriais (Howells \& Lopez, 2008; Ammentorp, Kofoed, \& Laulund, 2011, Coyne \& Gallagher, 2011, Croom et al., 2011, Forrest et al., 2012; Sleath et al., 2012; Di Battista et al., 2016; Davis, Burrows, Khallouq, \& Rosen, 2017).

Tornam-se relevantes, portanto, mais estudos sobre o contexto de assistência em pediatria que possam enfatizar a comunicação como uma política de incentivo à participação protagonista de pais ou cuidadores e também do usuário (neste caso a criança) como sujeito em seu próprio processo de desenvolvimento, uma estratégia de humanização aos cuidados que implica progressos diretos na qualidade da atenção em saúde da criança e preventivamente do adulto (Forrest et al., 2012; Maree, Parker, Kaplan, \& Osdthuizen, 2016). Estudos revelam que crianças a partir de quatro anos já compreendem informações básicas sobre autocuidados, identificam localização de órgãos e descrevem sintomas, têm dúvidas sobre a enfermidade, mencionam comportamentos promotores de saúde e descrevem implicações emocionais de um tratamento, elementos estes moderados pela idade e contexto socioeconômico da criança, história de contato com doenças em si ou em amigos/familiares e também pelas informações recebidas (Buckley \& Savage, 2010; Gordon et al., 2010; Knighting, Rowa-Dewar, Malcolm, Kearney, \& Gibson, 2010; Vatne, Slaughter \& Ruland, 2010; Gabarra \& Crepaldi, 2011; Forrest et al., 2012).

De modo especial, doenças crônicas infantis, como o câncer, requerem maior aprofundamento de estudos sobre comunicação e interações sob medida, tendo em vista preferências específicas por informação e demandas distintas em etapas diferentes do tratamento (Kästel, Enskär, \& Björk, 2011; Forrest et al., 2012; Di Battista et al., 2016; Haugen et al., 2016; Marre et al., 2016; Rodgers, Stegenga, Withycombe, Sachse, \& Kelly, 2016a; Rodgers et al., 2016b; Withycombre, Andam-Mejia, Dwyer, Slaven, Windt, \& Landier, 2016; Davis, et al., 2017). O câncer pediátrico corresponde a um grupo de mais de 100 doenças, caracterizadas pela multiplicação desordenada de células disfuncionais que prejudicam as funções do organismo (Instituto Nacional do Câncer -
INCA, 2018). Os tipos de câncer mais frequentes entre a população pediátrica são as variáveis deLeucemia (neoplasia de células hematopoiéticas), Tumores do Sistema Nervoso Central, Linfomas (neoplasias que acometem o sistema linfático), Neuroblastomas (neoplasias no sistema nervoso simpático), Tumores renais e hepáticos, Sarcomas ósseos e de partes moles (tumores em tecidos conectivos e de sustentação) e Retinoblastomas (tumores celulares na retina) (INCA, 2018).

0 tratamento em oncologia pediátrica é caracterizado pela repetição de procedimentos invasivos (punção lombar, punção venosa e exame de líquor), internações frequentes e longas, efeitos colaterais da quimioterapia (queda de cabelos, náuseas, vômitos, sangramentos), restrições a atividades sociais e de lazer, alterações na rotina profissional dos cuidadores, incertezas quanto à evolução e curabilidade do tratamento, medo de recaídas e cuidados específicos (atenção à higiene corporal e à saúde bucal, limpeza do ambiente doméstico e preparo de alimentos ), demandas potencialmente aversivas que exigem adaptação imediata da organização familiar e ajustes nos cuidados parentais (Kars, Duijnstee, Pool, Delden, \& Grypdonck, 2008, Vrijmoet-Wiersma et al., 2008; Forrest et al., 2012; Di Battista et al., 2016; Rodgers et al., 2016b).

No tratamento do câncer infantil, a qualidade da interação comunicativa e a recepção de informações sobre a doença, o estado físico da criança, os exames, procedimentos e efeitos colaterais são destacados pelos pais como ponto crucial ao longo do tratamento (Silva, 2000; Forrest et al., 2012; Di Battista et al., 2016; Haugen et al., 2016; Maree et al., 2016; Withycombre et al., 2016). Estudos sobre a comunicação na assistência ao câncer infantil enfatizam a qualidade da comunicação como moderadora da adaptação ao tratamento, tendo em vista a quantidade de informações recebidas na fase de diagnóstico que são assimiladas de forma gradual e subjetiva ao longo do tratamento (Zwaanswijk et al., 2011; Forrest et al., 2012; Maree et al., 2016; Rodgers et al., 2016a; 2016b; Withycombre et al., 2016). Ainda neste contexto, cabe configurar que a mobilização de questões emocionais tem potencialmente como consequência a criação de uma condição propícia a dificuldades na comunicação e na compreensão das orientações, enfatizando a necessidade de intervenções psicossociais que possam melhorar a interação durante consultas pediátricas (Forrest et al., 2012; Di Battista et al., 2016; Rodgers et al., 2016a).

Autores como Maree et al. (2016), Di Battista et al. (2016), Rodgers et al. (2016a) e também Davis et al. (2017) advogam a importância de estabelecer uma comunicação sob medida e afetuosa durante as consultas que visam o tratamento do câncer infantil, de modo a contemplar necessidades informa- 
tivas específicas de cada fase terapêutica, as preferências por informação e os estilos comunicativos de cada família, pois o diagnóstico geralmente corresponde a uma etapa complexa em função do choque inicial, implicando assimilação gradual e subjetiva das informações recebidas. Maree et al. (2016) destacam que o investimento na comunicação em contexto pediátrico é uma importante ferramenta de empoderamento ${ }^{1}$ dos pais, contribuindo sobremaneira para o sucesso terapêutico e a diminuição de perturbações psicológicas advindas das demandas de cuidados, muitas vezes complexas e aversivas.

Landier et al. (2016) destacam em seu estudo cinco elementos comuns a famílias que vivenciam um tratamento oncohematológico pediátrico e que devem ser cuidadosamente observados nos estudos e intervenções sobre comunicação: (1) a interação deve ser centrada na família e no paciente, de acordo com necessidades específicas de informação; (2) o diagnóstico é uma fase de choque e a família necessita de tempo para processar as informações e a nova realidade de tratamento, a fim de desenvolver um planejamento para organizar as novas demandas; (3) a interação e o acompanhamento devem ser realizados de modo interdisciplinar a partir de três pilares (diagnóstico/tratamento, manejo de demandas psicossociais e cuidados à criança); (4) a comunicação com a família e o paciente devem ocorrer de forma contínua durante todo o tratamento e não apenas na fase diagnóstica; (5) um contexto de suporte por parte da equipe de saúde é necessário para otimizar a assimilação das informações.

No âmbito brasileiro, destacamos os trabalhos de Silva (2000) e Zannon, Pereira, Arruda, Kohlsdorf e Rocha (2002): ambos estudaram os efeitos de uma entrevista com cuidadores pediátricos logoantes da consulta médica, com anotação de temas e dúvidas dos pais em formulário próprio anexado ao prontuário da criança. O médico, na consulta em seguida, poderia utilizar esta lista ao longo do atendimento, resultando em uma comunicação mais adequada às necessidades dos cuidadores. Essa intervenção foi denominada "pré-consulta comportamental" e apresentou resultados importantes, como melhor organização da comunicação durante consultas e aumento de temas a serem discutidos nas interações, além de maior empoderamento dos pais na relação terapêutica. Contudo, nestes dois estudos sobre a pré-consulta comportamental, não houve inclusão da criança na entrevista prévia às consultas nem a análise dos conteúdos estimados para o atendimento médico.

Tendo em vista as questões destacadas nesta Introdução, constitui objetivo do presente estudo analisar um procedimento de intervenção em pré-consulta comportamental, aplicado a cuidadores e pacientes pediátricos, relacionado à comunicação em consultas de acompanhamento em câncer infantil. Este trabalho é parte de uma pesquisa mais ampla sobre efeitos de entrevistas de pré-consulta, realizadas imediatamente antes de atendimentos médicos, sobre o repertório comunicativo de médicos, cuidadores e pacientes pediátricos. Neste artigo, são apresentados especificamente os conteúdos referidos nas entrevistas e uma análise prospectiva sobre estes temas ao longo do acompanhamento. Outros resultados da pesquisa, como por exemplo associações entre dados sociodemográficos e a comunicação, ou a permanência dos efeitos da intervenção em médio e longo prazo, podem ser obtidos em outros manuscritos e/ou a partir do contato com os autores.

\section{MÉTODO}

\section{Participantes}

Participaram deste estudo 15 díades cuidador-paciente. Critérios de inclusão envolveram a idade da criança (entre quatro e 13 anos de idade), idade dos cuidadores (maiores de 18 anos) e tempo de tratamento (no máximo seis meses desde o diagnóstico). Critérios de exclusão para o convite à participação corresponderam a crianças com comorbidades, em recaída da neoplasia ou que não responderam clinicamente ao tratamento, com sequelas cirúrgicas, dificuldades de ordem neurofisiológica relacionadas à verbalização ou díades que não dominavam o idioma português. A Tabela 1 apresenta dados sociodemográficos das díades acompanhadas neste trabalho.

\section{Instrumentos e materiais}

Foi desenvolvido para este estudo um protocolo para registro de pré-consulta comportamental, baseado nos estudos de Silva (2000) e Zannon et al. (2002), para descrever: (a) definição de cuidador e paciente sobre diagnóstico; (b) compreensão de cuidador e paciente sobre planejamento terapêutico; (c) dúvidas e assuntos estimados para a consulta; (d) dificuldades ou demandas relacionadas ao tratamento. Para abordar esses temas, um roteiro semi-estruturado para entrevista foi desenvolvido.

\section{Procedimento}

Em observância à Resolução número 196/96, do Ministério da Saúde, este estudo foi submetido ao Comitê de Ética em Pesquisa da Fundação de Ensino e Pesquisa em Ciências da Saúde da Secretaria de Estado de Saúde do Distrito Federal - FEPECS, aprovado em 05 de outubro de 2009, sob protocolo 301/09, parecer 289/2009. Cuidadores das díades que concordaram em integrar a pesquisa foram convidados a ler e assinar o Termo de Consentimento Livre e Esclarecido, bem como foi obtido consentimento verbal audiogravado das crianças.

como por exemplo idade dos cuidadores, tipo de diagnóstico, 


\section{W'INTERACÃO EM IF PSICOLOGIA}

Tabela 1. Caracteristicas das diades participantes.

\begin{tabular}{|c|c|}
\hline Características das Díades & Frequência \\
\hline \multicolumn{2}{|l|}{ Idade das crianças } \\
\hline 4 a 6 anos incompletos & 6 \\
\hline 6 a 8 anos incompletos & 3 \\
\hline 8 a 10 anos incompletos & 2 \\
\hline 10 a 12 anos & 4 \\
\hline MÉDIA (DP) & 7,74 anos $(3,25)$ \\
\hline \multicolumn{2}{|l|}{ Escolaridade das crianças } \\
\hline Creche ou maternal & 7 \\
\hline $1^{\text {a. }}$ a $3^{\text {a. }}$ série & 5 \\
\hline $4^{\text {a. }}$ série em diante & 3 \\
\hline \multicolumn{2}{|l|}{ Diagnóstico } \\
\hline Leucemias ou linfomas & 9 \\
\hline Tumores sólidos & 6 \\
\hline \multicolumn{2}{|c|}{ Tempo de tratamento ao início do procedimento } \\
\hline Até um mês & 5 \\
\hline Entre um e dois meses & 6 \\
\hline Entre dois e quatro meses & 2 \\
\hline Entre quatro e seis meses & 2 \\
\hline MÉDIA (DP) & 1,84 mês(1,47) \\
\hline \multicolumn{2}{|l|}{ Parentesco dos cuidadores } \\
\hline Mães & 13 \\
\hline Pais & 2 \\
\hline \multicolumn{2}{|l|}{ Idades dos cuidadores } \\
\hline Até 30 anos & 5 \\
\hline 31 a 40 anos & 8 \\
\hline Acima de 41 anos & 2 \\
\hline MÉDIA (DP) & 34,2 anos(7,34) \\
\hline \multicolumn{2}{|l|}{ Escolaridade dos cuidadores } \\
\hline Ens. Fundamental até $4^{\text {a. }}$ série & 1 \\
\hline Ens. Fundamental até $8^{\text {a. }}$ série & 3 \\
\hline Ensino Médio & 7 \\
\hline Ensino Superior & 4 \\
\hline \multicolumn{2}{|l|}{ Estado civil dos cuidadores } \\
\hline Casados/união estável & 9 \\
\hline Solteiros & 4 \\
\hline Divorciados & 2 \\
\hline \multicolumn{2}{|l|}{ Procedência } \\
\hline Distrito Federal & 12 \\
\hline Outros Estados (GO, MG, MT) & 3 \\
\hline \multicolumn{2}{|l|}{ Irmãos da criança em tratamento } \\
\hline Filho único & 2 \\
\hline Um ou dois irmãos & 9 \\
\hline Mais de dois irmãos & 4 \\
\hline \multicolumn{2}{|l|}{ Renda mensal familiar } \\
\hline Menos de 1 salário mínimo & 6 \\
\hline Entre 1 e 4 salários mínimos & 7 \\
\hline Mais de 4 salários mínimos & 2 \\
\hline
\end{tabular}

Em seguida, cada díade foi entrevistada individualmente enquanto aguardava por cada consulta médica na sala de espera. Dúvidas, demandas e assuntos referidos pelas díades foram registradas em um protocolo de pré-consulta, correspondente a uma folha de papel A4 com campos específicos para cada tema, que foi anexada à capa do prontuário da criança para ser utilizada na consulta em seguida como um indicador para os médicos sobre itens a serem incluídos na consulta (dúvidas, assuntos estimados, dificuldades e demandas).

As díades foram acompanhadas de forma contínua ao longo de três ou quatro consultas em sequência, a depender do esquema terapêutico individual, possibilitando análise de mudanças e/ou permanências nos itens indicados nas préconsultas ao longo deste período. 0 tempo médio empregado na entrevista de pré-consulta correspondeu a $17 \mathrm{~m} 02 \mathrm{~s}$ $(D P=6,34)$, com mínimo de $5 \mathrm{~m} 55 \mathrm{~s}$ e máximo $35 \mathrm{~m} 25 \mathrm{~s}$. Todas as consultas foram gravadas em áudio para posterior transcrição e análise de conteúdo segundo Bardin (1977).

\section{RESULTADOS}

Serão apresentados a seguir os resultados sobre modificações na quantidade e conteúdos dos assuntos referidos nas pré-consultas. Na Figura 1 é apresenta a quantidade de itens referidos ao longo de pré-consultas das díades acompanhadas ao longo de três ou quatro atendimentos. Houve padrão irregular da distribuição de itens indicados nas entrevistas de pré-consulta para crianças e cuidadores, com maior quantidade de itens mencionados pelos cuidadores. Crianças das díades 2, 9 e 10 (idades respectivamente 5, 4 e 7 anos) não indicaram dúvidas e não foi possível verificar tendências de aumento ou diminuição de itens indicados por estas crianças. Apenas para díades 7, 12 e 15 (idades respectivamente 13, 11 e 13 anos) houve relativo decréscimo em dúvidas e assuntos estimados ao longo das consultas, tendência também verificada para dificuldades e demandas de crianças nas díades 1, 3, 6 e 14 (idades respectivamente 8, 9, 10 e 11 anos). Crianças das díades 11 e 12 (idades respectivamente 4 e 11 anos) indicaram crescentes relatos de dificuldades e demandas entre as últimas entrevistas.

Considerando dúvidas e assuntos estimados para a consulta por parte dos cuidadores, houve tendência à diminuição de itens ao longo das entrevistas para díades 1, 2 , 6, 11 e 15; porém, houve aumento destes itens para díades 4, $5,7,9$ e 14. Os dados sociodemográficos de cada conjunto de díades - tanto aquelas que aumentaram quanto diminuíram os itens listados na pré-consulta - não apresentam particularidades comuns que pudessem estar diretamente relacionadas a esse aumento ou diminuição, 


\section{N"INTERACÃO EM ETH PSICOLOGIA}

renda mensal, estado civil ou escolaridade do cuidador.

Cuidadores das díades 12 e 14 aumentaram a referência a dificuldades e demandas ao longo dos atendimentos, porém houve diminuição desta categoria para díades 1, 3, 4, 5, 6, 7 e 8, com distribuições irregulares nas demais díades, dados que também não guardam relação direta com características
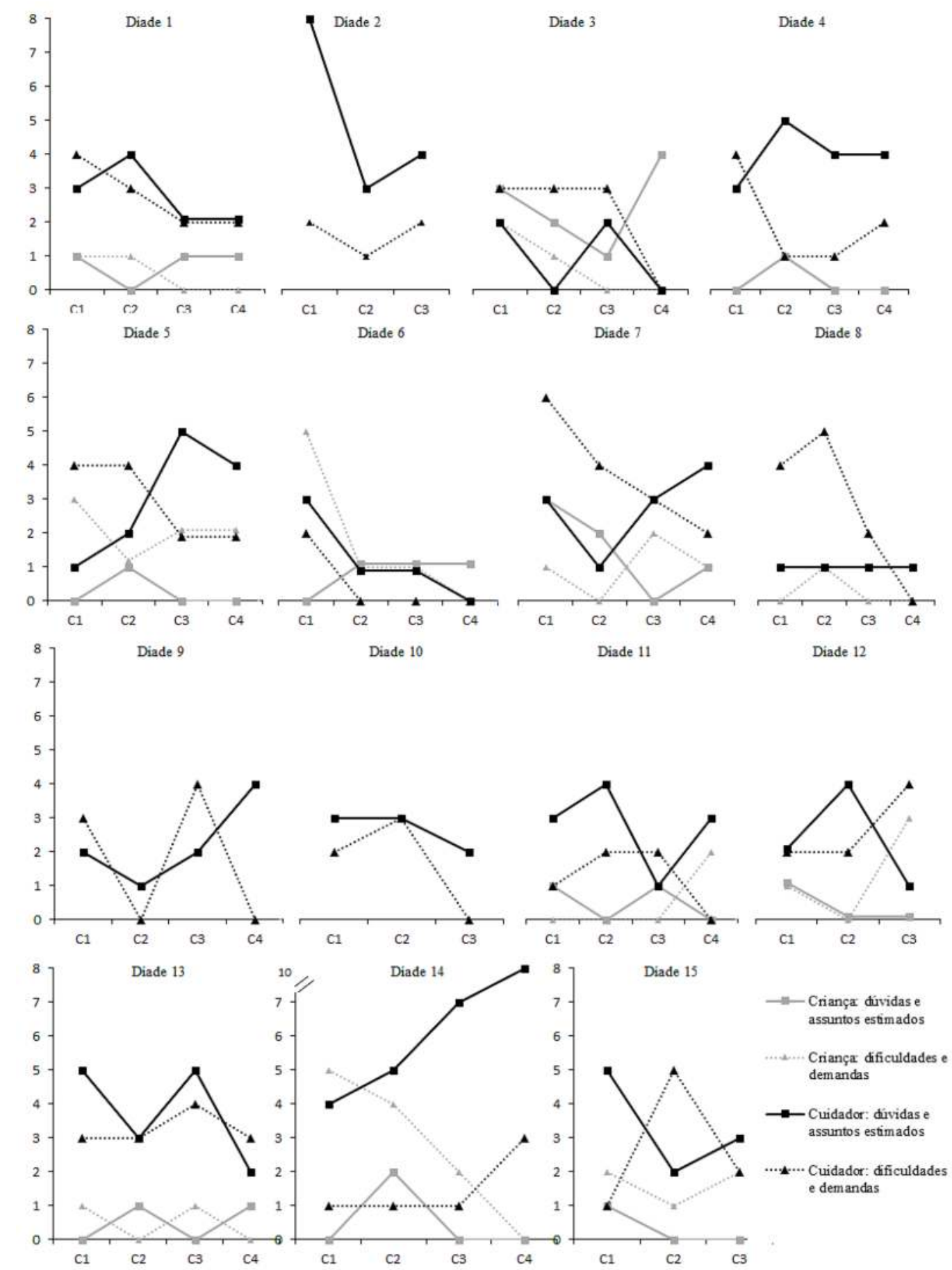

Figura 1. Quantidade e tipo de dúvidas ou demandas referidas nas pré-consultas. 


\section{- 4 I INTERACÃO EM LF PSICOLOGIA}

sociodemográficas. É possível que esses resultados indiquem necessidades diferentes de informação por parte de crianças e cuidadores ao longo do tratamento, dependendo por exemplo da resposta clínica do paciente e também de contextos individuais. $\mathrm{Na}$ Tabela 2 é indicado de modo decrescente o conteúdo referido pelas crianças nas pré-consultas.

A maior parte de dúvidas ou demandas esteve relacionada a exames, internações ou procedimentos $(\Sigma=18)$, assim como recomendações de cuidados $(\Sigma=10)$. Questões sobre atividade escolar, planejamento terapêutico e alimentação também apresentaram frequências notáveis (respectivamente, $\Sigma=9, \Sigma=8, \Sigma=8$ ). Em menores incidências, destacam-se questões sobre efeitos colaterais e sequelas $(\Sigma=5)$ e fundamentos da neoplasia ou tratamento $(\Sigma=5)$, contudo destacamse baixas ocorrências de dúvidas sobre sintomas ou intercorrências e preocupações sobre uso de outros remédios ( $\Sigma=5$ e $\Sigma=1$, respectivamente). Na Tabela 3 serão apresentados os relatos referidos pelas crianças nas pré-consultas e a análise prospectiva sobre a inclusão destes relatos ao longo das consultas.

Na Tabela 3 (em apêndice) são indicados resultados relevantes em relação à comunicação com crianças durante consultas. Houve demandas e dúvidas que, não incluídas na interação, foram novamente referidas em pré-consultas subsequentes (díades 1, 4 e 5, respectivamente 8,5 e 5 anos de idade), relacionadas à compreensão do diagnóstico e dificuldades com exames ou procedimentos. Contudo, crianças de diversas díades referiram itens permanentes ao longo de algumas consultas, mesmo que tais demandas fossem incluí- das na comunicação (díades 3, 5, 6, 11 e 12 respectivamente $9,5,10,4$ e 11 anos de idade). Esses assuntos estiveram relacionados à duração do tratamento, dificuldades emocionais ou comportamentais, preocupações com cirurgia ou exames, atividade escolar, dúvidas sobre alimentação e recomendações de cuidados, higiene ou lazer. Esses dados sugerem, por um lado, necessidades de informação distintas ao longo de etapas diferentes do tratamento ou ainda dificuldades relativas à própria comunicação, que dificulta a assimilação das informações. Por outro lado, houve também temas que, uma vez discutidos, não foram referidos posteriormente, como os relacionados a atividades escolares, recomendações de cuidados, higiene ou lazer e planejamento terapêutico.

Destacam-se alguns relatos referidos pelas crianças. Houve aspectos relacionados a fundamentos do tratamento e efeitos colaterais, como nos exemplos: "por que não posso sair ou ir à escola?"; "Bactrim causa mau hálito?"; "por que tenho cansaço após quimioterapia?"; "pra que servem os remédios?"; "o que é leucemia?"; "por que o cabelo demora a crescer?" e "como descobriram a leucemia?". Algumas dúvidas sobre recomendações de cuidados e higiene são ressaltadas: "do que posso brincar sem ficar cansado para a quimioterapia?" e "pode fazer natação?". Houve também demandas relacionadas a aspectos emocionais: "triste com cabelo caindo" e "acredita que familiares e equipe não estão sendo sinceros sobre a duração do tratamento". Na Tabela 4 são apresentados os temas indicados pelos cuidadores nas pré-consultas.

Houve concentração de dúvidas sobre sintomas ou manejo de intercorrências $(\Sigma=29)$, dificuldades sobre atividade profissional ou suporte familiar $(\Sigma=28)$ e efeitos colaterais/uso

\section{Tabela 2. Temas indicados pelos pacientes nas pré -consultas}

\begin{tabular}{ccccc}
\hline Temas & \multicolumn{3}{c}{ Consultas } \\
\cline { 2 - 5 } & 1 & 2 & 3 & 4 \\
\hline Dúvidas ou preocupação com exames, internações ou procedimentos & 9 & 3 & 2 & 4 \\
Dúvidas/demandas sobre recomendações de cuidados & 2 & 1 & 4 & 3 \\
Dúvidas ou preocupação sobre atividade escolar & 4 & 3 & 2 & - \\
Dúvidas ou preocupação sobre planejamento terapêutico & 3 & 4 & - & 1 \\
Dúvidas/demandas sobre recomendações para alimentação & 3 & 3 & 2 & - \\
Dúvidas ou preocupação sobre efeitos colaterais e seqüelas & 1 & 1 & 2 & 1 \\
Dúvidas sobre fundamentos da neoplasia ou tratamento & 3 & 2 & - & - \\
Dúvidas sobre sintomas ou intercorrências & 2 & - & 1 & 2 \\
Dúvidas sobre uso de outros remédios & - & - & 1 & -
\end{tabular}

Nota As Tabelas 3 e 5 encontram-se em apêndice. 


\section{- 4 I INTERACÃO EM LF PSICOLOGIA}

de medicação ( $\Sigma=26)$. Destaca-se também a quantidade de queixas sobre comportamentos da criança $(\Sigma=26)$ e dúvidas ou demandas sobre recomendações de cuidados $(\Sigma=25)$, bem como questões sobre planejamento terapêutico $(\Sigma=21)$. Em seguida, houve incidência relevante de dúvidas sobre alimentação $(\Sigma=18)$, demandas sobre resultados de exames e procedimentos $(\Sigma=18)$ e questões sobre atividade escolar $(\Sigma=17)$, além de preocupações sobre sequelas ou recaídas $(\Sigma=12)$. Em menor incidência, os cuidadores indicaram dúvidas sobre fundamentos da neoplasia ou tratamento $(\Sigma=9)$, dificuldades em práticas educativas $(\Sigma=8)$, demandas relativas a relatórios $(\Sigma=7)$ e dificuldades sobre organização do serviço de saúde $(\Sigma=4)$. A Tabela 5 (em apêndice) apresenta os relatos indicados pelos cuidadores nas pré-consultas e a análise prospectiva sobre a inclusão destes relatos ao longo das consultas. São apresentados dados interessantes sobre a comunicação de dúvidas e demandas dos cuidadores em consultas pediátricas. Houve itens que, não incluídos inicialmente no atendimento, foram referidos em consultas subsequentes (díades 1, 4, 7, 9, 10 e 14): demandas relativas a recomendações de cuidados, higiene ou lazer, queixas sobre comportamento da criança, preocupação com recaídas, dificuldades com serviço de saúde, dificuldades com alimenta- ção e vacinação, dúvidas sobre planejamento terapêutico ou cirúrgico, questões sobre uso de medicação não relacionada ao tratamento e dificuldades com outros filhos. As características socio-demográficas desses cuidadores são bastante distintas e nenhuma similaridade é destacada em relação a variáveis que possam subsidiar os dados apresentados. Contudo, todos os cuidadores, à exceção das díades 3, 8, 14 e 15, indicaram permanência de dúvidas e demandas contínuas, mesmo com discussão destas em consulta.

Algumas dessas demandas que permanecem presentes nos atendimentos, mesmo com sua inclusão na comunicação, naturalmente sofrem modificações ao longo de etapas diferentes de tratamento, tais como dúvidas sobre resultados de exames, sintomas da criança, planejamento da cirurgia, uso de medicação e demandas por relatórios diversos. Também foram repetidas demandas referentes à adaptação ao tratamento,processo que se estende por semanas e meses, como a adaptação da rotina, dificuldades em práticas educativas parentais e lidar com outros filhos, queixas sobre comportamentos da criança ou lidar com expectativas da família.

No entanto, é necessário ressaltar a permanência de dúvidas sobre questões básicas relacionadas ao tratamento on-

Tabela 4. Temas indicados pelos cuidadores nas pré -consultas

\begin{tabular}{ccccc}
\hline Temas & \multicolumn{3}{c}{ Consultas } \\
\cline { 2 - 5 } Dúvidas sobre sintomas ou manejo de intercorrências & 1 & 2 & 3 & 4 \\
Dificuldades sobre atividade profissional e/ou suporte familiar & 9 & 10 & 7 & 3 \\
Dúvidas sobre efeitos colaterais ou uso de medicação & 11 & 8 & 9 & - \\
Queixas sobre comportamentos da criança & 8 & 9 & 8 & 1 \\
Dúvidas/demandas sobre recomendações de cuidados & 9 & 6 & 5 & 6 \\
Dúvidas ou demandas sobre planejamento terapêutico & 8 & 5 & 5 & 7 \\
Dúvidas/demandas sobre recomendações para alimentação & 6 & 7 & 3 & 5 \\
Demandas sobre resultados ou realização de exames e procedimentos & 7 & 4 & 5 & 2 \\
Dúvidas ou preocupação sobre atividade escolar & 5 & 5 & 6 & 2 \\
Dúvidas ou preocupação sobre sequelas ou recaídas & 6 & 4 & 5 & 2 \\
Dúvidas sobre fundamentos da neoplasia ou tratamento & 6 & 3 & 1 & 2 \\
Dificuldades em práticas educativas parentais relacionadas ao tratamento & 3 & 4 & - & 1 \\
Requerimento de relatórios diversos & 5 & 2 & 1 & 1 \\
Dúvidas ou dificuldades sobre organização do serviço de saúde & 1 & 2 & - & 1 \\
\hline
\end{tabular}

Nota As Tabelas 3 e 5 encontram-se em apêndice. 


\section{-3* INTERACÃO EM LF PSICOLOGIA}

cohematológico, tais como recomendações de cuidados, higiene e lazer, uso de medicação não relacionada ao tratamento, manejo de atividades escolares e necessidade de restrições alimentares. Por outro lado, houve demandas discutidas nos atendimentos que não foram referidas em préconsultas posteriores, como as relacionadas a medicação, lidar com efeitos colaterais, atividades escolares, recomendações de cuidados, higiene ou lazer, efeitos colaterais e sequelas ou recaídas.

Alguns relatos referidos pelos cuidadores são destacados, especialmente em relação a fundamentos da neoplasia e tratamento: "leucemia é câncer?" e "o que causa a leucemia?"; "por que não pode tomar quimioterapia quando sangue está baixo?" e também "pra que serve a quimioterapia? Por que a quimioterapia tem efeitos colaterais?"; "pode usar anti-inflamatório e antibiótico para tratar o tumor?" e ainda "este tumor é de criança ou de idoso?". Falhas no uso de medicação também foram questões detectadas nas entrevistas de pré-consulta, bem como dúvidas sobre remédios e intercorrências, conforme relatos: "pode tomar suplemento alimentar?" e ainda "a partir de quantos graus é febre?" ou "pode usar chás e complementos?".

Houve dúvidas sobre sequelas ("quimioterapia pode ter sequelas no funcionamento hormonal?" e "criança vai voltar ao normal?") e perguntas sobre cuidados e higiene, como nos exemplos: "pode ir para a piscina?"; "precisa usar máscara?"; "que roupas deve usar no frio e no calor?" e ainda "precisa conferir temperatura e pressão arterial todo dia?" ou "no retorno à escola, a sala de aula será normal?". Houve dúvidas importantes sobre práticas educativas parentais, exemplificadas nos relatos "criança não comunica sintomas aos pais" ou "deve conversar com criança sobre a perda visual? Como?" e também "comportamento nervoso da criança quando mãe sai para trabalhar influencia o tratamento?".

\section{DISCUSSÃO}

Considerando os dados apresentados neste estudo, alguns aspectos interessantes são ressaltados. Especificamente no tratamento oncohematológico pediátrico, o uso de pré-consultas pode se mostrar relevante em função das exigências terapêuticas e da quantidade de informações recebidas na fase diagnóstica e assimiladas gradualmente, contribuindo para que cuidadores e crianças possam obter informações sob medida e de acordo com demandas de cada etapa terapêutica, conforme destacado como relevante na literatura (Kästel et al., 2011; Forrest et al., 2012; Di Battista et al., 2016; Haugen et al., 2016; Marre et al., 2016; Rodgers et al., 2016a; Rodgers et al., 2016b; Withycombre et al., 2016; Davis, et al., 2017).
O procedimento de pré-consulta facilita a abordagem sob medida a dúvidas e demandas específicas que caracterizam o tratamento oncohematológico, conforme evidenciado pelas maiores quantidades de assuntos específicos listados nas entrevistas tanto de crianças quanto de cuidadores. Considerando os temas indicados pelas crianças, houve prevalência de demandas sobre recomendações relacionadas a cuidados em higiene e alimentação, exames, internações ou procedimentos invasivos e fundamentos da neoplasia. Os cuidadores referiram majoritariamente assuntos sobre fundamentos da neoplasia, planejamento terapêutico, recomendações de cuidados, higiene e alimentação, manejo de intercorrências e dificuldades sobre atividade profissional ou suporte sociofamiliar. Esses assuntos também são apontados pela literatura como as principais demandas durante o diagnóstico e ao longo do tratamento do câncer infantil (Kars et al., 2008, Vrijmoet-Wiersma et al., 2008; Gabarra \& Crepaldi, 2011; Forrest et al., 2012; Di Battista et al., 2016; Rodgers et al., 2016b).

Os dados supracitados, em associação com a análise sobre mudanças ou permanências de assuntos estimados ao longo do atendimento, enfatizam o procedimento de pré-consulta como um processo especialmente contínuo que possibilita a cuidadores e crianças indicar temas específicos de seu interesse de acordo com diferentes etapas do tratamento, estilos subjetivos de comunicação e preferências individuais por informação, aspectos que vêm sendo enfatizados pela literatura como cruciais na comunicação pediátrica (Zwaanswijk et al., 2011; Forrest et al., 2012; Di Battista et al., 2016; Maree et al., 2016; Rodgers et al., 2016a; 2016b; Withycombre et al., 2016).

Em comparação com os estudos de Silva (2000) e Zannon et al. (2002), que também empregaram entrevistas de pré-consulta, destaca-se que esse procedimento enfatiza o empoderamento de cuidadores na comunicaçãoao estimular uma lista de assuntos estimados para a consulta médica. Por outro lado, o presente estudo entrevistou também os pacientes pediátricos, o que contribuiu para a inclusão de crianças na interação terapêutica, aspecto sublinhado pela literatura como imprescindível à comunicação eficiente em pediatria (Rotenberg et al., 2008, Vaknin \& Zisk-Rony, 2010; Gabarra \& Crepaldi, 2011; Forrest et al., 2012). O procedimento executado no presente estudo também esteve fundamentado nas proposições de Landier et al. (2016), ao promover uma interação centrada na família, com comunicação gradual de assuntos estimados sob demanda e com a provisão de informações de forma continuada, possivelmente promovendo suporte social à família e ao paciente.

Um aspecto importante ressaltado nos dados obtidos se refere à necessidade de compreender por que alguns temas ou dúvidas permanecem ao longo das consultas, indepen- 


\section{H INERACÄOEM ET PSICOLOGIA}

dentemente de terem sido debatidos ou não na interação comunicativa. Esse dado é coerente com outros estudos na literatura, que indicam que as informações são assimiladas de modo gradual ao longo do tempo (e, portanto, necessitam de repetição), tendo em vista a grande quantidade de demandas abruptas ao diagnóstico, as intensas adaptações familiares exigidas pelo tratamento oncohematológico pediátrico e a mobilização emocional provocada pelo adoecimento (Buckley \& Savage, 2010; Gordon et al., 2010; Knighting et al., 2010; Vatne et al., 2010; Gabarra \& Crepaldi, 2011; Forrest et al., 2012).

Sobre as limitações da presente pesquisa, destacamos a variabilidade nas características das díades, de modo especial o tempo de tratamento e as idades das crianças, elementos cruciais para contextualizar a comunicação em pediatria. Mesmo sabendo que uma amostra mais homogênea inviabilizaria a coleta de dados em tempo hábil, é sugerido que esta condição seja aprofundada em pesquisas futuras; além disso, a ampliação da amostra seria condição relevante para promover maior consistência dos dados, aspecto também enfatizado como proposta para outros estudos nesta temática. Trabalhos multicêntricos, do mesmo modo, são sugestão pertinente para avaliar a comunicação em contextos de saúde distintos. Mais estudos tornam-se necessários para avaliar especificamente os efeitos do procedimento de pré-consulta em longo prazo, bem como outras modalidades de intervenção que possam tornar a comunicação pediátrica mais eficiente.

\section{CONTRIBUIÇÃO DE CADA AUTOR}

M. K. foi responsável pela conceitualização, coleta de dados, análise de dados e redação do manuscrito; A. L. C. J. contribuiu com a conceitualização, supervisão na análise de dados e ajustes na redação do manuscrito.

\section{DECLARAÇÃO DE CONFLITOS DE INTERESSE}

Os autores declaram que não há conflitos de interesse no manuscrito submetido

\section{REFERÊNCIAS}

Ammentorp, J., Kofoed, P.E., \& Laulund, L.W. (2011). Impact of communication skills training on parents perceptions of care: Intervention study. Journal of Advanced Nursing, 67(2), 394-400. http://dx.doi.org/10.1111/j.1365 2648.2010.05475.x
Bardin, L. (1977). Análise de Conteúdo. Lisboa: Edições 70.

Buckley, A. \& Savage, E. (2010). Preoperative information needs of children undergoing tonsillectomy. Journal of Clinical Nursing, 19, 2879-2887. http://dx.doi.org/10.1111/j. 1365-2702.2010.03273.x

Coyne, I. \& Gallagher, P. (2011). Participation in communication and decision-making: Children and young people's experiences in a hospital setting. Journal of Clinical Nursing, 20, 2334-2343. http://dx.doi.org/10.1111/j.13652702.2010.03582.x

Croom, A., Wiebe, D.J., Berg, C.A., Lindsay, R., Donaldson, D., Foster, C., Murray, M., \& Swinyard, M.T. (2011). Adolescent and parent perceptions of patient-centered communication while managing type 1 diabetes. Journal of Pediatric Psychology, 36(2), 206-215. http://dx.doi.org/10.1093/ jpepsy/jsq072

Davis, J., Burrows, J.F., Khallouq, B.B., \& Rosen, P. (2017). Predictors of patient satisfaction in pediatric oncology. Journal of Pediatric Oncology Nursing, 34(6), 435-438. https:// doi.org/10.1177/1043454217717239

Di Battista, A., Dupuis, L.L., Cassidy, M., Portwine, C., Johnston, D.L., Silva, M.P., Sung, L., \& Barrera, M. (2016). Parent attributions about child symptoms related to cancer therapy. Journal of Pediatric Oncology Nursing, 34(1), 44-50. https://doi.org/10.1177/1043454215628002

Fisher, M.J. \& Broome, M.E. (2011). Parent-provider communication during hospitalization. Journal of Pediatric Nursing, 26, 58-69. http://dx.doi.org/10.1016/j.pedn.2009.12.071

Forrest, C.B., Bevans, K.B., Tucker, C., Riley, A.W., Ravens-Sieberer, U., Gardner, W., \& Pajer, K. (2012). Commentary: The Patient-Reported outcome measurement information system (PROMIS) for children and youth: Application to pediatric Psychology. Journal of Pediatric Psychology, 37(6), 614-621. https://doi.org/10.1093/jpepsy/jss038

Gabarra, L.M. \& Crepaldi, M.A. (2011). A comunicação médico-paciente pediátrico-família na perspectiva da criança. Psicologia Argumento, 29(65), 209-218. https://doi.org/ 10.7213/rpa.v29i65.20335

Gordon, B.K., Jaaniste, T., Bartlett, K., Perrin, M., Jackson, A., Sandstrom, A., Charleston, R., \& Seehan, S. (2010). Child and parental surveys about pre-hospitalization information provision. Child: Care, health and development, 37(5), 727733. http://dx.doi.org/10.1111/j.1365-2214.2010.01190.x

Haugen, M.S., Landier, W., Mandrell, B.N., Sullivan, J., Schwartz, C., Skeens, M.A., \& Hockenberry, M. (2016). Educationg families of children newly diagnosed with cancer. Journal of Pediatric Oncology Nursing, 33(6), 405-413. https://doi.org/10.1177/1043454216652856 


\section{H INERACÄOEM ET PSICOLOGIA}

Howells, R. \& Lopez, T. (2008). Better communication with children and parents. Paediatrics and Child Health, 18(8), 381-385. http://dx.doi.org/10.1016/j.paed.2008.05.007

Instituto Nacional do Câncer [INCA] (2018). Informações sobre o câncer pediátrico. Recuperado de http://www.inca.gov.br

Kästel, A., Enskär, K. \& Björk, O. (2011). Parent's views on information in childhood cancer care. European Journal of Oncology Nursing, 15, 290-295. https://doi.org/10.1016/ j.ejon.2010.10.007

Kars, M. C., Duijnstee, M. S. H., Pool, A., Delden, J. J. M., \& Grypdonck, M. H. F. (2008). Being there: Parenting the child with acute lymphoblastic leukaemia. Journal of Clinical Nursing, 17(12), 1553-1562. http://dx.doi.org/10.1111/ j.1365-2702.2007.02235.x

Knighting, K., Rowa-Dewar, N., Malcolm, C., Kearney, N., \& Gibson, F. (2010). Children's understanding of cancer and health behaviors. Child: Care, health and development, 289-299.

Landier, W., Ahern, J., Barakat, L. P., Bhatia, S., Binger, K. M., Bondurant, P. G., Cohn, S. L., Dobrozi, S. K., Haugen, M., Herring, R. A., Hooke, M. C., Martin, M., Murphy, K., Newman, A. R., Rodgers, C. C., Ruccione, K. S., Sullivan, J., Weiss, M., Withycombe, J., Yasui, L., \& Hockenberry, M. (2016). Patient/family education for newly diagnosed pediatric oncology partients: Consensus recommendations from a children's oncology groups expert panel. Journal of Pediatric Oncology Nursing, 33(6), 422-431. https://doi.org/ $10.1177 / 1043454216655983$

Maree, J. E., Parker, S., Kaplan, L., \& Osdthuizen, J. (2016). The information needs of south African parents of children with cancer. Journal of Pediatric Oncology Nursing, 33(1), 9-17. https://doi.org/10.1177/1043454214563757

Rodgers, C. C., Laing, C. M., Herring, R. A., Tena, N., Leonardelli, A., Hockenberry, M., \& Hendricks-Ferguson, V. (2016). Understanding effective delivery of patient and family education in pediatric oncology. Journal of Pediatric Oncology Nursing, 33(6), 432-446. https://doi.org/ $10.1177 / 1043454216659449$

Rodgers, C. C., Stegenga, K., Withycombe, J. S., Sachse, K., \& Kelly, K. P. (2016). Processing information after a child's cancer diagnosis - How parents learn. Journal of Pediatric Oncology Nursing, 33(6), 447-459. https://doi.org/ $10.1177 / 1043454216668825$

Rotenberg, K. J., Cunningham, J., Hayton, N., Hutson, L., Jones, L., Marks, C., Woods, E., \& Betts, L. R. (2008). Development of a children's trust in general physicians scale. Child: Care, health and development, 34(6), 748-756. https://doi.org/10.1111/j.1365-2214.2008.00872.x
Silva, M. M. (2000). Consulta pediátrica: efeito de procedimentos instrucionais sobre os temas tratados. (Tese de Doutorado). Universidade de Brasília, Brasília.

Sleath, B., Carpenter, D. M., Slota, C., Williams, D., Tudor, G., Yeatts, K., Davis, S., \& Ayala, G.X. (2012). Communication during pediatric asthma visits and self-reported asthma medication adherence. Pediatrics, 130(4), 1-7. http:// dx.doi.org/10.1542/peds.2012-0913

Vaknin, O. \& Zisk-Rony, R.Y. (2010). Including children in medical decisions and treatments: Perceptions and practices of healthcare providers. Child: Care, Health and Development, 37(4), 533-539. http://dx.doi.org/10.1111/j.13652214.2010.01153.x

Vatne, T. M., Slaughter, L., \& Ruland, C. M. (2010). How children with cancer communicate and think about symptoms. Journal of Pediatric Oncology Nursing, 27(1), 24-32. http://dx.doi.org/10.1177/1043454209349358

Vrijmoet-Wiersma, C. M. J., Van Klink, J. M. M., Kolk, A. M., Koopman, H. M., Ball, L. M., \& Egeler, R. M. (2008). Assessment of parental psychological stress in pediatric cancer: A review. Journal of Pediatric Psychology, 33(1), 1-13. http://dx.doi.org/10.1093/jpepsy/jsn007

Withycombre, J. S., Andam-Mejia, R., Dwyer, A., Slaven, A., Windt, K., \& Landier, W. (2016). A comprehensive survey of institutional patient/family educational practices for newly diagnosed pediatric oncology patients - A report frm the Children's Oncology Group. Journal of Pediatric Oncology Nursing, 33(6), 414-421. https://doi.org/ $10.1177 / 1043454216652857$

Zannon, C. M. L. C., Pereira, R. M. G., Arruda, P. M., Kohlsdorf, M., Rocha L. E. P. (2002). Pré-consulta comportamental em reumatologia pediátrica: identificando problemas e promovendo aliança para o tratamento. Revista Brasileira de Reumatologia, 79, 14-19.

Zwaanswijk, M., Tates, K., van Dulmen, S., Hoogerbrugge, P. M., Kamps, W. A., Beishuizen, A., \& Bensing, J. M. (2011). Communicating with child patients in pediatric oncology consultations: A vignette study on child patient's, parent's, and survivor's communication preferences. Psycho-Oncology, 20, 269-277. http://dx.doi.org/10.1002/pon.1721

Data de submissão: 25 de fevereiro de 2017 Primeira decisão editorial: 05 de dezembro de 2017 Aprovação: 15 de março de 2018

\footnotetext{
1 Segundo Maree et al. (2016), termo referente a participação mais ativa dos pais na relação terapêutica, implicando melhor autonomia dos cuidadores, comportamentos mais assertivos e inclusão de demandas próprias na interação.
} 


\section{APÊNDICES}

Tabela 3 Conteúdo referido pelas crianças e análise prospectiva sobre a inclusão de itens na consulta

\begin{tabular}{|c|c|c|c|c|c|}
\hline \multicolumn{2}{|r|}{ Temas } & \multicolumn{4}{|c|}{ Consultas } \\
\hline & & 1 & 2 & 3 & 4 \\
\hline \multirow[t]{2}{*}{ Díade 1} & Rever compreensão: diagnóstico & $\mathrm{X}$ & $\mathrm{X}$ & & \\
\hline & Quanto dura esta internação? & & $x$ & & \\
\hline \multirow[t]{10}{*}{ Díade 3} & Rever recomendações: cuidados & $X^{*}$ & & & \\
\hline & Duração estimada do tratamento & $X^{*}$ & $X^{*}$ & & \\
\hline & Chateado por não ir à escola & $X^{*}$ & & & \\
\hline & Por que sente dores na barriga? & $X^{*}$ & & & \\
\hline & Por que não pode ir à escola? & $X^{*}$ & & & \\
\hline & Chateado com queda do cabelo & & $X^{*}$ & & \\
\hline & Por que se sente mal no calor? & & & $X^{*}$ & \\
\hline & Queixas sobre sintomas & & & & $X^{*}$ \\
\hline & Por que tem cansaço após quimioterapia? & & & & $X^{*}$ \\
\hline & Bactrim causa mau hálito? & & & & $X^{*}$ \\
\hline \multicolumn{2}{|c|}{ Do que pode brincar sem ficar cansado para a quimioterapia? } & & & & $X^{*}$ \\
\hline \multirow[t]{3}{*}{ Díade 4} & Dúvida:diagnóstico & $\mathrm{X}$ & $X^{*}$ & & \\
\hline & Duração tratamento & $X^{*}$ & & & \\
\hline & Há restrições a atividades? & & & & $X^{*}$ \\
\hline \multirow[t]{3}{*}{ Díade 5} & Dúvidas sobre diagnóstico & $\mathrm{X}$ & & & \\
\hline & Dificuldades com exames ou remédios & $x$ & $x$ & & $X^{*}$ \\
\hline & Dificuldades emocionais (nervosismo) & & & $X^{\star}$ & $X^{*}$ \\
\hline \multirow[t]{16}{*}{ Díade 6} & Dificuldades emocionais & $X^{\star}$ & & & \\
\hline & Rever recomendações de atividades & $X^{*}$ & & & \\
\hline & Dificuldade com exames & $X^{*}$ & $X^{*}$ & & \\
\hline & Chateada por não ir à escola & $X^{*}$ & & $X^{\star}$ & \\
\hline & Dificuldades familiares & $X^{*}$ & & & \\
\hline & Planejamento terapêutico & & $X^{*}$ & & \\
\hline & Pode usar pomada para hematomas? & & & $X^{*}$ & \\
\hline & Pode fazer natação? & & & & $X^{\star}$ \\
\hline & Quando vai desinchar? & $X^{\star}$ & & & \\
\hline & O que são os caroços? & $X^{*}$ & & & \\
\hline & Quando voltar à escola? & $X^{*}$ & $x$ & & \\
\hline & Dificuldades emocionais & $X^{*}$ & & & $X^{*}$ \\
\hline & Há restrição a atividades & & & $x$ & \\
\hline & Quer fazer regime por causa do Decadron & & & $X^{*}$ & \\
\hline & Duração do tratamento & & & & $X^{*}$ \\
\hline & Pra que servem os remédios? & & $\mathrm{X}$ & & \\
\hline
\end{tabular}




\section{*3' INTERACÃO EM \\ FT PSICOLOGIA}

Tabela 3. Conteúdo referido pelas crianças e análise prospectiva sobre a inclusão de itens na consulta (continuação)

\begin{tabular}{|c|c|c|c|c|c|}
\hline \multicolumn{2}{|r|}{ Temas } & \multicolumn{4}{|c|}{ Consultas } \\
\hline & & 1 & 2 & 3 & $\overline{4}$ \\
\hline Díade 7 & Quando voltará à escola? & & $X^{\star}$ & & \\
\hline \multirow[t]{3}{*}{ Díade 8} & Dificuldade com exames & $\mathrm{X}$ & & & \\
\hline & Quanto tempo até comer normalmente? & & $X^{*}$ & & \\
\hline & Triste com cabelo caindo & & & $X^{*}$ & \\
\hline \multirow[t]{7}{*}{ Díade 11} & Rever diagnóstico & $X^{*}$ & & & \\
\hline & Rever recomendações alimentares & $X^{*}$ & $X^{*}$ & & \\
\hline & Dificuldades com exames e cuidados & $X^{*}$ & $X^{\star}$ & & \\
\hline & Chateado por não ir à escola & & $X^{*}$ & & \\
\hline & Chateado por não poder passear & & $X^{*}$ & $X^{*}$ & \\
\hline & Rever planejamento terapêutico & & $X^{*}$ & & \\
\hline & Rever recomendações de cuidados & & & $X^{*}$ & \\
\hline \multirow[t]{4}{*}{ Díade 12} & Dúvidas sobre alimentação & $X^{\star}$ & $X^{*}$ & & \\
\hline & Preocupação com cirurgia & $X^{\star}$ & & $X^{*}$ & \\
\hline & Planejamento terapêutico & $x$ & & & \\
\hline & Dúvidas sobre cuidados & & & $X^{\star}$ & \\
\hline \multirow[t]{2}{*}{ Díade 13} & Duração desta internação & $X^{*}$ & & & \\
\hline & Separação do pai durante internação & $x$ & & & \\
\hline \multirow[t]{3}{*}{ Díade 14} & Rever restrições a alimentação & & & $\mathrm{X}$ & \\
\hline & Rever recomendações de cuidados & & & $x$ & \\
\hline & Chateada por não ir à escola & & & $\mathrm{X}$ & \\
\hline \multirow[t]{3}{*}{ Díade 15} & Quando poderá liberar ingestão de sal? & $\mathrm{X}$ & & & \\
\hline & Pode comer danoninho? & & & $X^{\star}$ & \\
\hline & Dificuldades em exames e procedimentos & & & & $\mathrm{x}$ \\
\hline
\end{tabular}

Nota: $\mathrm{X}$ sinalizados no protocolo de pré -consulta, * Incluídos na consulta 


\section{WIIITERACÃO EM ET PSICOLOGIA}

Tabela 5. Conteúdo referido por cuidadores e análise prospectiva sobre a inclusão de itens na consulta

\begin{tabular}{|c|c|c|c|c|c|}
\hline \multicolumn{2}{|r|}{ Temas } & \multicolumn{4}{|c|}{ Consultas } \\
\hline & & 1 & 2 & 3 & 4 \\
\hline \multirow[t]{15}{*}{ Díade 1} & Rever orientações: cuidados e atividades & $\mathrm{X}$ & & & $X^{*}$ \\
\hline & Queixas sobre comportamento da criança & $x$ & & & $\mathrm{X}$ \\
\hline & Dificuldades em procedimentos & $x$ & & & \\
\hline & Medo de recaídas & $x$ & $X^{*}$ & & \\
\hline & Discutir a possibilidade de cateter & $X^{*}$ & & & \\
\hline & Como o organismo está reagindo? & $x$ & & & \\
\hline & Rever restrições alimentares & & $\mathrm{X}$ & & \\
\hline & Como lidar com a queda de imunidade? & & $X^{*}$ & & \\
\hline & Duração estimada do tratamento & & $X^{*}$ & & \\
\hline & Dúvidas sobre uso de medicação & & $X^{\star}$ & $X^{*}$ & \\
\hline & Para que serve a Mercaptina? & & $X^{\star}$ & & \\
\hline & Pode retirar Nausedron na farmácia? & & $X^{*}$ & & \\
\hline & Poderá ir à escola este ano? & & & $X^{\star}$ & \\
\hline & Dificuldade sobre duração do tratamento & & & $x$ & \\
\hline & Como será a próxima fase? & & & $X^{*}$ & $X^{*}$ \\
\hline \multicolumn{2}{|c|}{ A quimioterapia pode ter sequelas no funcionamento hormonal? } & & & $X^{\star}$ & \\
\hline & Qual periodicidade da punção medular? & & & & $X^{\star}$ \\
\hline & Requerimento de relatório & & & & $X^{\star}$ \\
\hline \multirow[t]{9}{*}{ Díade 2} & Queixas sobre sintomas da criança & $X^{*}$ & & $X^{\star}$ & \\
\hline & Dúvidas sobre a cura & $\mathrm{x}$ & & & \\
\hline & Há restrições de atividades? & $X^{*}$ & & & \\
\hline & Dúvidas sobre frequência escolar & $X^{*}$ & & & \\
\hline & Requerimento de relatório & & $X^{*}$ & $X^{\star}$ & \\
\hline & Dificuldade de adaptação da rotina & & $X^{*}$ & $X^{*}$ & \\
\hline & Falha no uso de medicação & & $\mathrm{X}$ & & \\
\hline & Suporte conjugal insatisfatório & & & $X^{*}$ & \\
\hline & Rever diagnóstico & $X^{*}$ & & & \\
\hline \multirow[t]{6}{*}{ Díade 3} & Quais são as causas da leucemia? & $X^{*}$ & & & \\
\hline & Dúvidas sobre alimentação & $X^{*}$ & & & \\
\hline & Criança tem comido muito & $X^{\star}$ & & & \\
\hline & Dúvida: planejamento terapêutico & $X^{*}$ & & & \\
\hline & Queixas sobre sintomas da criança & & $X^{\star}$ & & \\
\hline & Discutir possibilidade de catéter & & & $X^{*}$ & \\
\hline
\end{tabular}




\section{H NTERAC̄öEM

Tabela 5. Conteúdo referido por cuidadores e análise prospectiva sobre a inclusão de itens na consulta (continuação)

\begin{tabular}{|c|c|c|c|c|}
\hline \multirow[t]{2}{*}{ Temas } & \multicolumn{4}{|c|}{ Consultas } \\
\hline & 1 & 2 & 3 & 4 \\
\hline Rever recomendações a atividades & $X^{\star}$ & $\mathrm{X}$ & & \\
\hline Vai recuperar a habilidade visual? & $X^{\star}$ & & & \\
\hline Atenção constante a sintomas é difícil & $\mathrm{X}$ & & & \\
\hline Criança fala os sintomas aos pais & $X^{\star}$ & $x$ & $x$ & $x$ \\
\hline Demora no diagnóstico & $x$ & $\mathrm{X}$ & & \\
\hline Retorno à escola & $X^{\star}$ & & & \\
\hline Solicitação de atestado & $X^{\star}$ & $X^{*}$ & $X^{*}$ & \\
\hline Dúvidas sobre alimentação & & $X^{*}$ & & \\
\hline Medicação não relativa a tratamento & & $X^{*}$ & & \\
\hline Queixas sobre sintomas da criança & & & $X^{\star}$ & $X^{*}$ \\
\hline Pode ir para a piscina? & & & & $X^{*}$ \\
\hline Díade 5 Deve conversar com criança sobre a perda visual? Como? & & $X^{*}$ & & \\
\hline Pode tomar suplemento alimentar? & $X^{\star}$ & & & \\
\hline A partir de quantos graus é febre? & $X^{*}$ & & & \\
\hline Como será a cirurgia? & $X^{\star}$ & $X^{*}$ & $X^{*}$ & \\
\hline Queixas: comportamento da criança & $X^{\star}$ & $X^{*}$ & $\mathrm{x}$ & \\
\hline Dificuldades profissionais & $X^{\star}$ & & & \\
\hline Por que a criança tem perdido peso? & $X^{\star}$ & & & \\
\hline Precisa doação de sangue da família? & $X^{\star}$ & & & \\
\hline O cabelo vai cair? & $X^{\star}$ & & & \\
\hline Deve explicar mais para a criança sobre o tratamento? & $X^{\star}$ & & & \\
\hline Poderá voltar à escola? & $X^{\star}$ & & & \\
\hline Queixas sobre sintomas da criança & & $X^{\star}$ & $X^{\star}$ & \\
\hline Planejamento terapêutico & & $X^{*}$ & & \\
\hline Resultados de exames & & $X^{*}$ & $X^{*}$ & \\
\hline Pode dar vitaminas para apetite? & & & $X^{*}$ & \\
\hline Queixas sobre nervosismo da criança & $X^{\star}$ & $X^{*}$ & $X^{*}$ & \\
\hline Criança não quer comer & $X^{\star}$ & $X^{*}$ & & \\
\hline Triste por não saber como explicar restrições escolares à criança & $X^{\star}$ & $X^{\star}$ & & \\
\hline Preocupada com outros filhos & $X^{\star}$ & & & \\
\hline O que causa a leucemia? & $X^{\star}$ & & & \\
\hline Medo de expor a criança em passeios & & $X^{*}$ & & \\
\hline Planejamento terapêutico & & $X^{\star}$ & & \\
\hline Pode usar remédios para gripe? & & & $X^{*}$ & \\
\hline
\end{tabular}




\section{W'INTERACÃO EM ET PSICOLOGIA}

Tabela 5. Conteúdo referido por cuidadores e análise prospectiva sobre a inclusão de itens na consulta (continuação)

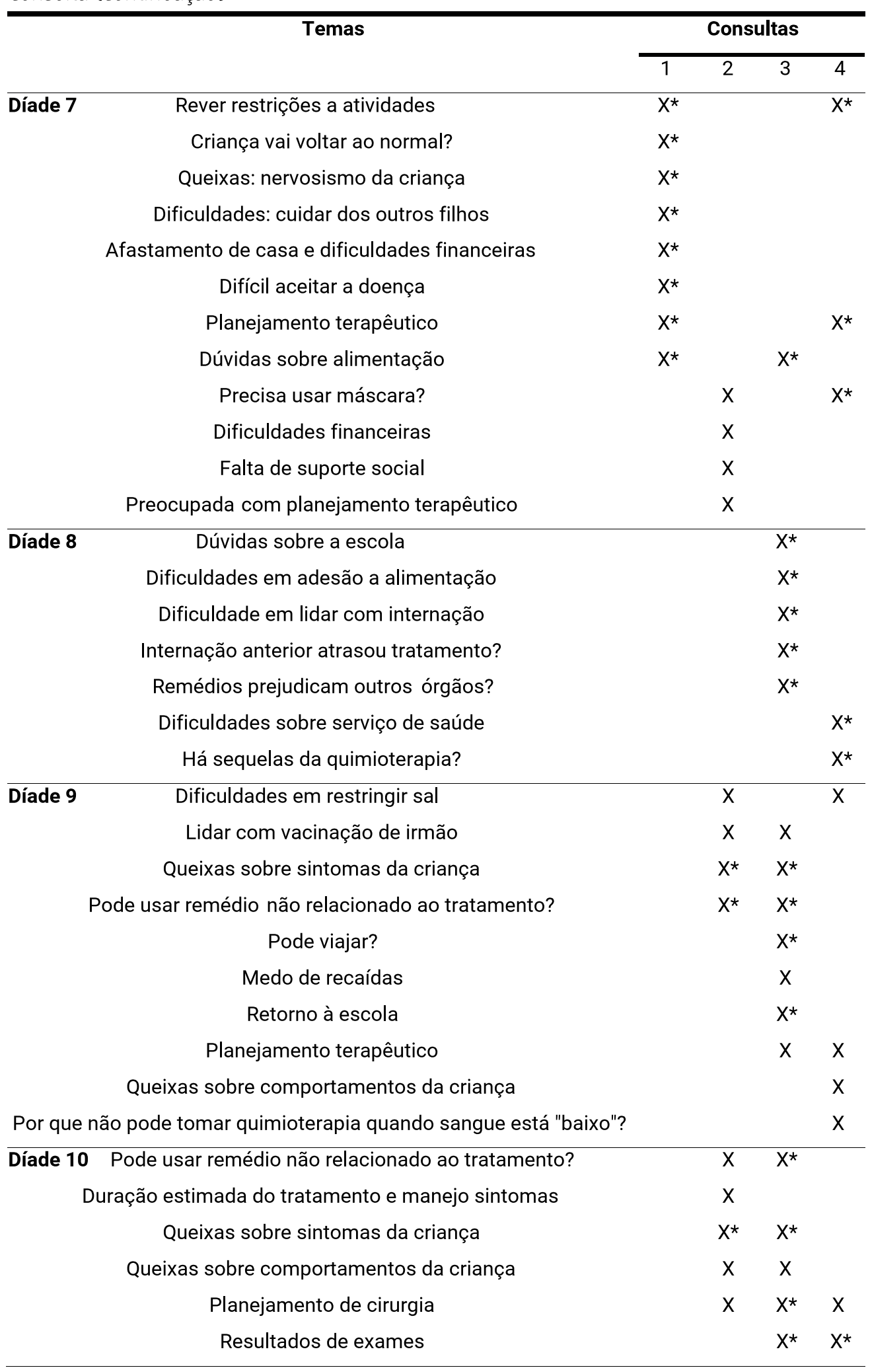




\section{H NTERAC̄öEM

Tabela 5. Conteúdo referido por cuidadores e análise prospectiva sobre a inclusão de itens na consulta (continuação)

\begin{tabular}{|c|c|c|c|c|}
\hline \multirow[t]{2}{*}{ Temas } & \multicolumn{4}{|c|}{ Consultas } \\
\hline & 1 & 2 & 3 & 4 \\
\hline Rever restrições alimentares & $X^{*}$ & & $X^{\star}$ & \\
\hline Dificuldades com exames & $\mathrm{X}$ & & & \\
\hline Dificuldades: lidar com expectativas da família & $X^{*}$ & $X^{*}$ & $X^{\star}$ & \\
\hline Medo sobre recaída & $X^{*}$ & $\mathrm{X}$ & & \\
\hline Dúvidas sobre atividade escolar & $X^{\star}$ & & & $X^{*}$ \\
\hline Dúvidas sobre alimentação & $X^{*}$ & & & \\
\hline Dificuldades emocionais & & $X^{\star}$ & & \\
\hline Planejamento terapêutico & & $X^{*}$ & $X^{*}$ & \\
\hline Dificuldades no serviço de saúde & & $X^{*}$ & & \\
\hline Queixas:comportamento da criança & & & $X^{*}$ & $X^{\star}$ \\
\hline Pode viajar? & & & $X^{\star}$ & \\
\hline Quando vai desinchar? & & & $X^{\star}$ & \\
\hline Vai precisar aumentar a proteção e isolamento? & & & $x$ & \\
\hline Dificuldade: adesão à medicação & & & & $X^{\star}$ \\
\hline Discutir uso do cateter & & & & $X^{\star}$ \\
\hline Dificuldades alimentares & & & & $X^{\star}$ \\
\hline É melhor explicar internação pra criança ou não? & & & & $X^{\star}$ \\
\hline Rever restrições a passeios & $X^{*}$ & & $\mathrm{X}$ & \\
\hline Por que ocorrem efeitos colaterais? & $\mathrm{X}$ & & & \\
\hline Pode utilizar remédios caseiros e chás? & $X^{*}$ & $X^{*}$ & $\mathrm{x}$ & \\
\hline Dificuldade em deixar outros filhos em casa & $\mathrm{X}$ & & $\mathrm{X}$ & \\
\hline Como manejar efeitos colaterais? & & $\mathrm{x}$ & & \\
\hline Queixas sobre sintomas da criança & & $\mathrm{X}$ & $x$ & \\
\hline Sintomas são normais, há risco de desmaio? & & $\mathrm{X}$ & & \\
\hline Falhas no uso de medicação & & & $X^{\star}$ & \\
\hline Díade 15 Preocupação exacerbada com higiene e restrições & $\mathrm{X}$ & & $\mathrm{X}$ & \\
\hline Medo de recaídas & $\mathrm{X}$ & & & \\
\hline Planejamento terapêutico & $X^{*}$ & & & \\
\hline Pode usar remédio não relacionado ao tratamento? & $\mathrm{X}$ & & & \\
\hline Rever liberação de sal em função do uso de Decadron & & $\mathrm{X}$ & & \\
\hline Resultados de exames & & & $X^{\star}$ & \\
\hline Dificuldades de adaptação da rotina do cuidador ao tratamento & & & $\mathrm{x}$ & \\
\hline Pode ter contato com quem tomou vacina? & & & & $X^{\star}$ \\
\hline Solicitação de relatório & & & & $X^{\star}$ \\
\hline Queixas sobre sintomas da criança & & & & $X^{\star}$ \\
\hline
\end{tabular}

Nota: $\mathrm{X}$ sinalizados no protocolo de pré -consulta, * Incluídos na consulta 